\title{
Redes sociales y su utilidad en la educación ambiental promoción y divulgación informal
}

\section{Social networks and their utility in environmental education promotion and informal disclosure}

\author{
Belkys J. Guzmán' \\ belkysguzman19@hotmail.com \\ belkys.juliana.guzman@gmail.com \\ ${ }_{1}^{1}$ Universidad Pedagógica Experimental Libertador. Instituto Pedagógico de Caracas, Venezuela \\ ${ }^{2}$ Ministerio de Educación y Ministerio de Defensa, Venezuela

\section{Emma L. López ${ }^{2}$} \\ lopezemma@hotmail.com
}

Recibido 5 de julio 2019| Revisado ago-sep 2019 | Publicado 01 de octubre 2019

\section{RESUMEN}

La accesibilidad de las personas a la información, gracias a las redes sociales se puede convertir en saberes, conocimiento y participación ciudadana. El presente artículo tiene por objetivo difundir la promoción y divulgación de la Educación Ambiental Informal en la formación del ciudadano a través de la Redes Sociales Digitales. Generando espacios de propagación de contenidos que dinamicen el acceso al conocimiento de "saberes" sobre acontecimientos significativos en materia ambiental que sirvan de base en la formación del ciudadano y produzca un cambio de actitud hacia el ambiente. Estos aspectos muestran un gran desarrollo en la enseñanza y aprendizajes para todos los productos y sus contenidos, con elementos interactivos y didácticos para el desarrollo de sus actitudes y aptitudes en el entorno escolar.

Palabras clave TIC, TAC, Redes Sociales y Educación Ambiental

\section{ABSTRACT}

The accessibility of people to information, thanks to social networks, can become wisdom, knowledge and citizen participation. This article has the objective to spread the promotion and dissemination of Informal Environmental Education, in citizen training through the Digital Social Networks, generating spaces for the propagation of contents that increase the access to the knowledge of wisdom about significant environmental events that serve as a basis for citizen training and produce a change in attitude towards the environment. These aspects show a great development in teaching and learning for all products and their contents, with interactive and didactic elements for the development of their attitudes and aptitudes in the school environment.

Key words: ICT, ACT, Social Networks and Environmental Education 


\section{INTRODUCCIÓN}

En el siglo XXI, la era digital ha desarrollado una revolución virtual en la vida del ser humano, mediante el cual se ha visto en la necesidad de ir adaptándose a los cambios que la tecnología de los nuevos tiempos ha desarrollado y ha mejorado el día a día del ciudadano en todas las dimensiones de la colectividad por medio de una interconexión local, regional y global (Krotoski, 2012).

Hoy se dan ciertas prácticas sociales y culturales que hasta hace poco tiempo no habían impactado en las formas de comunicación. Los jóvenes están "conectados" de forma continua. La participación en redes sociales como Facebook, grupos de Whatsapp, foros de temas de su interés y otras formas de participación hace que se evidencie una actividad intensa con diferentes tecnologías. (González y otros, 2019).

En consecuencia, la sociedad se ha beneficiado al tener acceso a una amplia cobertura de dispositivos con tecnologías de avanzada, que despliegan diversas opciones de uso y eleva la calidad de vida de los usuarios, lo cual evidencia un nivel de impacto importante en la sociedad. Entre estas opciones, se destacan el uso de las Redes Sociales digitales, las cuales conforman las tecnologías de avanzada que Medina y De Haro (2010) considera "son servicios Web especializados en permitir relaciones de algún tipo entre las personas" (p. 38), que tienen marcadas diferencia con respecto a las tecnologías tradicionales. Al hacer referencia a éstos últimos, Sevillano (2005) las describe como:

El límite entre lo nuevo y lo viejo de las Tecnologías de la Información y la Comunicación (TIC), se puede establecer en función de la tecnología en la cual se apoyan, siendo por lo general, las tecnologías tradicionales unidireccionales en el proceso de la comunicación, jerárquicas, colectivas y rígidas, sin proceso de feed-back, y con pocas posibilidades de elección de itinerarios comunicativosformativos-informativos por los receptores (p. 273)

Con las TIC es fundamental para el ámbito digital en el aprendizaje, pero juntos con el uso de las TAC estas representa un gran desarrollo en las enseñanza y aprendizajes para todos los productos y sus contenidos, con elementos interactivos y didácticos para el 1 desarrollo de sus actitudes y aptitudes en el entorno escolar pero para todos esto, es necesario que muestren sus habilidades usando su concentración cuando el docente este explicando su teoría y participen con motivación a este elemento, (Fernández, 2019).

Un ejemplo de lo señalado con anterioridad lo constituyen las redes sociales digitalizadas que han creado un tejido de interrelaciones de personas, instituciones o comunidades con intereses comunes, las redes sociales se han convertido en el fenómeno de mayor extensión, pues han incursionado en todos los ámbitos de la vida del humano, en la política, el deporte, el arte y el espectáculo, la cultura, salud, el mercado, la economía, en la educación y el ambiente.

\section{MÉTODO}

El presente estudio se refiere a una investigación de tipo documental basado en la revisión de fuentes bibliográficas relacionadas con al estado del arte de lo que diversos autores han aportado en relación con las redes sociales digitales, definiciones, ejemplos, sus tipos y su utilización en la promoción y difusión de la educación ambiental informal. En este marco se consideró la revisión de diversas 
investigaciones desarrolladas por diferentes autores entre las que figuran las que realizan este articulo entre muchos otros, para contrastar las percepciones individuales acerca del tópico y así identificar a posteriori la existencia o no de puntos de coincidencia y de descenso entre sus enfoques.

La investigación formó parte de un conjunto de estudios dirigidos a reconstruir teóricamente el objeto de investigación que se denomina: redes sociales y sus usos en la promoción y difusión de la educación ambiental. Sobre esta temática se han producido ya diversos trabajos, artículos y ponencias. Atendiendo a la amplitud y complejidad de dicho objeto se propone dilucidar progresivamente algunas de las claves que pueden ayudar a orientar la inserción pertinente de las redes sociales.

\section{RESULTADOS}

El mundo se encuentra compuesto de signos lingüísticos y tecnológicos que obligan a leer, entender y comunicarnos. En fin integrar las TIC propiciando que los docentes, utilicen la tecnología de una manera que les facilite los procesos de investigación, comprensión, síntesis y análisis de información, haciendo uso del Internet, plataformas Web 2.0 y 3.0, aplicaciones informáticas (Apps), redes sociales, Streaming (retransmisión de contenido multimedia), y de esta manera concretar en las TAC (Tecnologías del Aprendizaje y del Conocimiento). (Hobrayan, Carpio y Villagómez (2019).

En este sentido, las Redes Sociales superan las propiedades de las tecnologías tradicionales, en los siguientes aspectos, son: multidireccionales en el desarrollo de la comunicación, dinámicas, se puede obtener retroalimentación in situ, entre otros. Dichas redes se corresponden con una plataforma de avanzada calificada de segunda generación
(2G) denominada Web2.0, la cual permite optimizar la interacción entre los usuarios, al respecto Medina y De Haro (2010) señala que:

Web 2.0 hace referencia a las características sociales y de colaboración que ha adquirido Internet. Aparecen nuevos servicios que permiten a los usuarios interactuar entre sí y establecer una colaboración más allá de los simples mensajes o comentarios. Así pues, la Web 2.0 no es un cambio tecnológico, ni un avance informático. (p. 24)

Este tipo de plataforma determina el alcance comunicacional de las Redes Sociales, lo cual significa que en cualquier parte del mundo independientemente de la geografía y el horario, las personas pueden comunicarse con la única limitación que le dé su naturaleza personal. Al respecto, Ballestrini, Marcon, Morduchowicz y Sylvestre (2010) plantean que:

$$
\begin{aligned}
& \text { Las redes sociales son } \\
& \text { "comunidades virtuales". Es decir, } \\
& \text { plataformas de internet que } \\
& \text { agrupan a personas que se } \\
& \text { relacionan entre sí y comparten } \\
& \text { información e intereses comunes. } \\
& \text { Este es justamente su principal } \\
& \text { objetivo: entablar contactos con } \\
& \text { gente ya sea para reencontrarse } \\
& \text { con antiguos vínculos o para } \\
& \text { generar nuevas amistades (p. 3). }
\end{aligned}
$$

Para estos autores, las Redes Sociales conforman un conjunto de usuarios, quienes según sus posibilidades de dedicación, los motiva un común denominador como es: el construir, desarrollar, compartir información y conocimientos; pero lo más importante es que el usuario ha creado su espacio digital o huella social, que según Bonnelly (2011), "es la suma de los contactos personales y las relaciones digitales en las distintas redes 
sociales, multiplicado por el número de usuarios promedio de cada una de las redes a las que se encuentre conectado" (p. 15). Además, con contenido inédito en las Redes Sociales, de forma gratuita y con un espacio que le permite tener la potencialidad de llegar a multitudes de nuevos usuarios.

Las Redes Sociales digitales han sido adoptadas como recursos de comunicación de primera necesidad en este mundo globalizado, de tal manera que es importante su conocimiento y aplicación en el ámbito educativo. De Haro (2008), declara que "las Redes Sociales tienen el innegable valor de acercar el aprendizaje informal y el formal, ya que permiten al alumno expresarse por sí mismo, entablar relaciones con otros, así como atender a las exigencias propias de su educación" (p. 1).

Cabe señalar, que al tratar de relacionar a las Redes Sociales con el tema educativo, se hace necesario mencionar que en la educación se identifican tres tipos de ámbitos: el formal, el no formal y el informal siendo éste último, para efectos de este estudio, donde las Redes Sociales tienen su espacio pertinente.

$\mathrm{Al}$ respecto, la Coordinadora de ONG para el Desarrollo de España (2004), define la educación informal como aquella que:

La educación informal se adquiere en los diferentes espacios sociales e influye en el desarrollo personal y social de los individuos. En el ámbito informal se desarrolla la sensibilización y la educación es obtenida por medio, entre otros, de la combinación de la observación, la imitación y la emulación de miembros específicos de la sociedad. (p. 24).

La educación informal se desarrolla en el amplio espectro del entorno social del ser humano a través de diversos medios que coadyuvan con el aprendizaje, entre los que se mencionan a: la familia, los círculos sociales, las Organizaciones No Gubernamentales, los consejos comunales, los medios de comunicación, la televisión, la radio, internet, las Redes Sociales Analógicas, las Redes Sociales Digitales, entre otros. Es de hacer notar, que la concepción de las Redes Sociales digitales se ajusta dentro de las herramientas educativas recomendadas por la Agenda 21 (1992), promulgadas en la Conferencia de Río, referente al Capítulo 36 sobre el Fomento de la Educación, la Capacitación y la Toma de Conciencia, en el literal "H", donde se sugiere el uso de las TIC para promover la educación sobre el ambiente, por tanto:

Es necesario fortalecer... el
intercambio de información
mediante el mejoramiento de la
tecnología y los medios
necesarios para promover la
educación sobre el medio
ambiente y el desarrollo y la
conciencia del público. Los países
deberían cooperar entre sí y con
los diversos sectores sociales y
grupos de población para
preparar instrumentos
educacionales que abarquen
cuestiones e iniciativas regionales
en materia de medio ambiente y
desarrollo, utilizando materiales
y recursos de aprendizaje
adaptados a sus propias
necesidades (p. 2).

La preparación de materiales educativos concernientes a la Educación Ambiental, la cual es definida por Martínez (citado por Lárez y Carrero, 2003) en los siguientes términos:

Un proceso educativo, integral e interdisciplinario que considera al ambiente como un todo y que busca involucrar a la población en general en la identificación y 
solución de problemas a través de la adquisición de conocimientos, valores, actitudes y habilidades, la toma de decisiones y la participación activa organizada (p. 111).

Cabe considerar, que ese concepto es producto de una necesidad de cambio en las acciones humanas hacia el ambiente, tomando en cuenta el elevado índice de deterioro en el que se encuentra, el cual precisa de ser tratado desde lo educativo en todos los ámbitos: formal, no formal y en mayor medida en lo informal por su amplio alcance.

Sin embargo, el pronóstico de la situación ambiental es desalentador para las actuales y futuras generaciones, tal como lo señala Lacroix (1994 en López, 2016 p.6) cuando manifiesta que:

La preocupación ecológica
impone su obsesiva presencia en
todos los ámbitos, pues no hay
nadie que no sea consciente del
empobrecimiento de la capa de
ozono, del recalentamiento por el
efecto invernadero, de la
degradación del suelo y del medio
marino, del problema del agua y
de los residuos industriales, de la
deforestación, del agotamiento de
los recursos, de la
superpoblación, del abismo
económico entre los dos
hemisferios y de la inestabilidad
del mundo.

En este contexto, de por sí preocupante y aunado a la necesidad de mayor información sobre las consecuencias que estos problemas ocasionan en la mayoría de los sectores de la población, el deterioro del entorno irá en aumento ocasionando daños irreversibles en las condiciones de habitabilidad y poniendo en riesgo la vida en el planeta, si no se toman acciones que contrarresten el desgaste de los recursos naturales.
Por lo tanto, se hace indispensable que la ciudadanía tenga la información que requiere para conformar un nuevo orden social orientado hacia la protección y conservación del ambiente, desde lo sustentable y enmarcado dentro de la educación ambiental informal por medio de las Redes Sociales digitales, de manera que les permita hacer una adecuada participación y toma de decisiones.

La importancia de las Redes Sociales digitales radica en la potencialidad de la información expedita que las caracterizan como medios comunicativos entre personas de diferentes niveles sociales, culturales, con distintos intereses, preferencias, entre otros, los cuales han conformado una cultura comunicacional digital a nivel global.

En este aspecto, Bonnelly (2011) señala que:

las Redes Sociales... han roto
todos los esquemas de
crecimiento de audiencias para
colocarse hoy comor los
principales medios res de
comunicación de la historia, con
usuarios que superan las
poblaciones de la mayoría de los
países del mundo. Solo hay que
ver lo que tardaron algunos
medios en llegar a la cifra de 50
millones de usuarios: la radio,
treinta y ocho años; la televisión
lo logró en catorce años; Internet
Explorer de Microsoft, en cuatro,
mientras que Facebook agregó el
año pasado cien millones de
usuarios en apenas nueve meses
(p. 16).

En este mismo orden de ideas, la Unión Internacional de Telecomunicaciones de la ONU informó que existen 2300 millones de personas, aproximadamente un tercio de la población mundial, conectada a internet (Google, 2012, Octubre 11). 
A nivel Latinoamericano, en entrevista realizada a Carlos Jiménez, Gerente de Tendencias Digitales, expresó que el número de usuarios en internet alcanza la cifra de 231 millones (Suárez, 2012) caracterizado de la siguiente manera:

La conexión a internet es más cotidiana y más personal, pues más del $60 \%$ de usuarios se conectan desde el hogar. Más del $50 \%$ de usuarios de Internet en la región se conectan todos los días. Más del 20\% de usuarios de Internet se conectan a través de su dispositivo móvil en Latinoamérica (Cuerpo 1, p.9).

Cabe destacar, que entre las plataformas más usadas se encuentran Facebook con un $89 \%$, YouTube con un $85 \%$, Google con un $61 \%$, Twitter con un $41 \%$ y Skype con un $43 \%$, y en lo referente al Ranking de usos de Internet en la región se encuentra Chile en primer lugar, Venezuela en segundo y Argentina en tercero (op. cit).

La segunda tendencia es sobre el tema ambiental, donde sólo el 8,9\% manifestó interés y preocupación en el tópico, la misma pudiera servir de referencia sobre la necesidad de promocionar de forma enfática y con efecto multiplicador a la Educación Ambiental en la población, para tener mayor información, formación y generación de acciones más cónsonas con el entorno, de manera de contener el índice de deterioro. Esta situación, pudiera ser resultado de muchos factores, entre los que se encuentra, por una parte, que la Educación Ambiental formal no abarca toda la población, y en cuanto a la educación ambiental informal en lo que respecta a los medios de comunicación social, en el caso de la televisión con señal abierta, dentro de su programación requiere de una revisión de los contenidos por tener una visión distanciada de la temática ambiental como eje transversal.
Las Redes Sociales han contribuido con la cultura tecnológica en las sociedades. En el siglo XXI, la era cibernética, caracterizada por un surgimiento vertiginoso de la tecnología la cual ha elevado la eficiencia en todas las dimensiones de la sociedad a través de una interconexión a nivel global (Faundes y Aristizabal, 2001), marca el contexto histórico del ser humano, quien se beneficia de una amplia cobertura de tecnologías de avanzada inimaginables en el siglo pasado, las cuales han conformado una cultura tecnológica en la humanidad.

Es de hacer notar, que la diferencia en los tipos de TIC radica en el tipo de plataforma que la apoya; al respecto, Sevillano (2005) expresa:

El límite entre lo nuevo y lo viejo en las TIC, se puede establecer en función de la tecnología en la cual se apoyan, siendo por lo general las tecnologías tradicionales unidireccionales en el proceso de la comunicación, jerárquicas, colectivas y rígidas, sin proceso de feed-back, y con pocas posibilidades de elección de itinerarios comunicativosformativos-informativos por los receptores (p. 273)

En este sentido, cada época tiene sus adelantos científicos - tecnológicos en años anteriores las tecnologías estarían apoyadas con la Web 1.0 que se caracteriza por ser unidireccional, es decir, que van en línea directa del emisor al receptor sin tener la oportunidad de retroalimentar el hecho comunicacional. Sustituidas por las plataformas de avanzada denominada Web 2.0, calificada de segunda generación determina su alcance comunicacional por la potencialidad que la caracteriza en traspasar el tiempo y el espacio. Ya se habla de 3.0. En consecuencia, su aplicación se ha multiplicado de manera acelerada por su potencialidad en 
su función comunicativa, es decir, acorta las distancias $y$ se puede obtener una retroalimentación (feedback) en tiempo real. De igual manera, Beltrán y Castañeda (2004) manifiestan que:

Las redes sociales son como una comunidad de personas que, de modo formal o informal, ocasionalmente, a tiempo parcial o de forma dedicada, trabajan con un interés común y basan sus acciones en la construcción, el desarrollo y la compartición mutuos de conocimientos (p. 1).

Para estos autores las Redes Sociales conforman un conjunto de personas, quienes según sus posibilidades de dedicación los motiva un común denominador como es el construir, desarrollar y compartir intereses y conocimientos. Mientras que para Chópite (2014) las Redes Sociales representan a grupos de personas que se relacionan entre sí por interés sobre un tema 0 por tener afinidades específicos para López, 2016 cita a Prato y Viloria (2010) son sistemas que conforman relaciones con otros usuarios a quienes se les puede conocer o no en persona, por medio del cual se crean relaciones por diversas razones, ya sean personales, profesionales, entre muchas otras.

López (2016) hace referencia a Merodio (2010) quien las considera como herramientas que representan un hito en la evolución de las formas tradicionales de como el ser humano se comunica, que han trascendido por medio de nuevos canales con herramientas fundamentados en el conocimiento colectivo y la confianza ya que por medio de ellas ya que desde el punto de vista comercial tanto la empresa como los consumidores serian generadores de contenido de distinta índole, entre ellos de la educación ambiental.

\section{Tipos de Redes Sociales}

Las redes sociales digitales que operan en la Web se pueden clasificar según distintos criterios: a) dirigidas a todo tipo de usuario, con o sin una temática definida, b) que cambia en función de las relaciones entre personas, los contenidos compartidos o los eventos creados, c) por su plataforma tecnológica, lo importante es que los docentes deben desarrollar procesos de enseñanza $y$ aprendizaje que incluya contenidos educativos de manera formal y no formal de las redes sociales con y a través de ellas (Bucarelo y Zambrano, 2010).

Guzmán $(2005,2008,2013)$ señala que existen distintos tipos de Redes Sociales, en primer lugar se encuentran las que se caracterizan en las redes analógicas, en las cuales no se necesitan un dispositivo electrónico y otras redes digitales en las cuales se necesita un dispositivo electrónico como soporte, bien sea celulares, computadoras que dan la posibilidad de comunicarse con otros en cualquier lugar del mundo.

Las Redes analógicas o sociales Off-Line: Son redes formadas por grupos de personas con alguna relación en común, que se desarrollan sin sistemas electrónicos o informáticos conectados a la red como por ejemplo las familias, los grupos de seguridad de los barrios. Mientras que las Digitales o Redes sociales On-Line son las que tienen su origen y se desarrollan a través de medios electrónicos e informáticos.

Al respecto, Burgueños (2009), clasifica las redes en analógicas, las denomina también redes sociales Off-Line, y digitales o redes sociales On-Line, donde en las primeras, su característica base es que se desarrollan sin necesidad de una plataforma de circuitos electrónicos, por ejemplo, en las barriadas populares de Caracas se emplea el sonar de un pito para dar aviso de alerta en las 
comunidades ante una situación de riesgo. En las segundas, su característica principal y que las diferencia de las anteriores, es la necesidad de tener una plataforma de equipos electrónicos y digitales para su funcionamiento.

También pueden ser de tipo general como:

$>$ Personales

$>$ Temáticas

$>$ Profesionales

Las personales son aquellas que se componen de millones de usuarios en los que cada quien se empodera de su "espacio" dando su información personal acompañado con sus fotos, así como expresa cuáles son sus gustos y/o hobbies como la música, el cine, literatura, juegos, deportes entre otros, cada uno se puede relacionar con los demás de múltiples maneras como invitar a un contacto, buscar amigos, invitarlos a jugar, aunque la plataforma base es la internet de una manera $\mathrm{u}$ otra Facebook es una red social personal.

En cuanto a las Redes Temáticas son similares a las anteriores pero se diferencian en que conforman comunidades con intereses por un tema en especial, entonces comparten y desarrollan información asociada al tópico en cuestión, por ejemplo una red de cine, teatro, deportes, música, juegos, informativa, salud, belleza y una variedad de opciones tan interminable como los intereses de los seres humanos.

Por otra parte, están las redes profesionales las cuales presentan una variedad distintivas con respecto a las anteriores, ya que se dedican exclusivamente a lo relacionado con el ámbito laboral en todas sus dimensiones, desde poner en contacto a aquellos que ofrecen trabajo con los que lo buscan, así como crear grupos que estén relacionados con trabajos de cualquier nominación o pertenecientes a una empresa pública o privada.
Jaramillo (2011) expresa que hay dos clases de Redes Sociales que son: verticales y horizontales. Las verticales son instituidas por los propios internautas, entre ellas se encuentran: Ninq, Bigtent, SocialGo, Spruz, Mixxt, Shoutem, Twiducate y Edmodo. Las horizontales son las redes establecidas por programadores a las que se unen los internautas. Ejemplos de estas plataformas: Facebook, YouTube, Foursquare, MySpace, Metacafe, Jisko, Flickr, Friendfee, Google Buzz, Ketere, Hi5, Window Live Space, Picotea.com, Tuenti, Xing, Bebo, Friendster, Sonico, Wikipedia, Orkut, Badoo, MyFamilypedia y Boombang entre otras.

Chópite (2014) en relación a este tipo de redes señala que autores como Liberos y otros (2011) consideran que están enfocadas en audiencias menores en contenidos pero que cuentan con una gran proliferación de contenidos específicos como de maternidad, educación, religión, ambiente, deportes o sexualidad y Fernández (2010) las describe como aquellas que están concebidas sobre la base de un eje temático. Igualmente, tienen como objetivo congregar en torno a una temática definida a un colectivo determinado.

Las horizontales son las redes son aquellas que abarcan todo tipo de contenidos y son establecidas por programadores a las que se unen los internautas. Fernández (2010 citado por Chópite, 2014) señala que son "aquellas redes que están dirigidas a todo tipo de usuario y no presentan una temática específica. Además expone que éstas permiten la entrada y participación libre y genérica sin un fin determinado". Ejemplos de estas plataformas: Facebook, Youtube, Foursquare, MySpace, Metacafe, Jisko, Flickr, Friendfee, Google Buzz, Ketere, Hi5, Window Live Space, Picotea.com, Tuenti, Xing, Bebo, Friendster, Sonico, Wikipedia, Orkut, Badoo, MyFamilypedia y Boombang se encuentra la creación de sitios regionales de contacto 
entre usuarios para fomentar el acercamiento entre dichos miembros de la comunidad.

\section{Redes Sociales en el Ámbito Educativo}

Las Redes Sociales han sido adoptadas como recursos de comunicación de primera necesidad en este mundo globalizado. Ellas se corresponden con la Web 2.0, "una característica permite la interrelación de las personas, facilitando el dialogo y el aprendizaje colaborativo" (Sobrino, 2011). De tal manera, es importante su conocimiento como una herramienta de enseñanza virtual, entendida como el recurso que pueda apoyar en el ámbito educativo por medio de plataformas digitales (Sevillano, 2005), en especial en el ámbito de la Educación Ambiental informal la cual es derivada de una necesidad de cambio paradigmático en la relación de las acciones humanas hacia el entorno, y por lo tanto puede y debe ser promocionada por los mediadores $\mathrm{y}$ formadores informales a través de las Redes Sociales.

En el caso venezolano, autores tales como De Marco (2015), Guzmán (2005, 2008, 2013), Marquina (2011) manifiestan que son varios los esfuerzos realizados por el estado venezolano en la implantación de plataformas tecnológica en el campo educativo, desde la elaboración de la Normativa Legal que fundamenta la aplicación de las TIC, como el Plan TIC. A pesar de esto, De Marco (2008) expresa que algunos maestros y profesores no han descubierto el enorme potencial que encierra el uso de esta herramienta y los servicios educativos que ofrece, por falta de capacitación y formación. Eso también ocurre con la inserción de las Redes Sociales en el ámbito educativo, según Marquina (2011) se puede atribuir a diversos factores, tales como: ausencia de programas de actualización, pensum universitarios no actualizados, dificultad de los docentes de tener acceso a la modernización tecnológica, ausencia de estímulos, lo cual genera docentes no info- alfabetizados, tecnofobia, entre otros.

Para Guzmán (2013, 2018, 2019) la evolución ocurre con la llegada de la Web 2.0, ahora se trata de concebir los avances que tendrán la Web 3.0 y Web 4.0, se tienen expectativas de muchas posibilidades de unificación de información. Aunque estas herramientas Web 2.0 no estaban destinadas originalmente para su uso en el aula, pueden ser medios de aprendizaje extremadamente efectivos, tanto para el educación formal, no formal como la informal. Tiene una potencialidad inmensa al permitir que se estimulen todos los canales de percepción y además y los ciudadanos pueden interactuar cara a cara a través de dispositivos, lo que disminuye la soledad y permiten el aprendizaje cooperativo y también el constructivo.

Para esta misma autora (op. cit) está claro que la Web 2.0 tiene valor educativo real que los docentes gozan de aplicación en sus aulas y fomentan la interactividad y la participación, motivación y formación de los participantes. Con estos medios se incentiva el compromiso y el empoderamiento, propiciando una mayor participación activa de los estudiantes en el aula y en la realización de las tareas. Además ayudan a tomar disfrutar de sus propios proyectos independientemente de su estilo de aprendizaje y de qué tipo de inteligencia tenga más desarrollada.

Con el uso de las redes sociales se establece un compromiso por parte del alumno porque le corresponde moderar el mismo su aprendizaje, por medio de las ventajas que ofrecen estos recursos.

En efecto, las Redes Sociales pueden favorecer el desempeño de los formadores informales digitales en Educación Ambiental en el proceso educativo, cuando se les emplea como herramientas educativas virtuales que servirán de apoyo en la difusión, promoción y enseñanza de la Educación Ambiental. 
Es de hacer notar que en el ámbito educativo informal, no se ha hecho esperar la incorporación de las Redes Sociales, tales como Facebook, Twitter y YouTube, como estrategia de enseñanza y aprendizaje para los internautas, el cual se ha convertido en una revolución en el campo formativo las cuales se convierten en herramientas virtuales indispensables para lo educativo porque coadyuvarían en la divulgación de la información a nivel global, local y, además, en tiempo real.

Bajo esta perspectiva, la educación se dirige hacia la nueva docencia 2.0, la cual se orienta a obtener competencias, habilidades y conocimientos, en las comunidades virtuales instituidas, los cuales se convierten en los pilares fundamentales para la promoción del aprendizaje activo (De Haro, 2008).

Por su parte Rodríguez (2010) expresa que las Redes Sociales son espacios que existen en internet que les permiten a los usuarios encontrarse por un interés común, bien sea para compartir o para interactuar. Además coinciden que la base fundamental de una red social la interacción entre personas quienes de manera recíproca forman, construyen y comparten información de manera empática. Existen miles de páginas y grupos abiertos y cerrados relacionados con contenidos específicos o para el desarrollo de cursos, actividades o trabajos de equipos, líneas de investigación.

\section{Potencialidad de las Redes Sociales}

En el siglo XXI, las Redes Sociales han conformado una cultura tecnológica en las sociedades. Su aporte en todas las dimensiones del acontecer humano, las convierte en recursos imprescindibles para coadyuvar en elevar el nivel de su calidad de vida. Su estructura permite a los usuarios acceder a la información, incorporar datos y tomar decisiones. Se incrementa, en este caso, las posibilidades de actualización y vigencia de la información, determinar tendencias de eventos y hechos, que se pueden extraer inmediatamente y recibir mensajes a costos racionales, Por tanto es importante emplear la potencialidad en el ámbito de la Educación Ambiental,

El uso de las Redes Sociales se ha incrementado en los últimos años, existiendo una variedad de ellas a nivel mundial, sin embargo, de ese grupo se destacan las más populares y más utilizadas como son Twitter, Facebook, YouTube y LinkedIn, En este sentido, Medina y De Haro, (2010) señala que las características generales de las redes sociales permiten:

1. Mantener en contacto a las personas sin barreras de espacio ni tiempo.

2. La participación de un gran número de personas.

3. Desplegar un ambiente propicio de trabajo.

4. Optimizar la comunicación.

5. Inmediatez en la ubicación de personas.

6. La comunicación es sencilla: de forma pública por medio del muro (término acuñado a una forma de dejar un aviso, un memo o una comunicación) y de manera privada mediante mensajería.

7. Una mejor observación en la comunicación e interacción participantetutor.

8. Conformar grupos de: trabajo, intereses, hobbies, entretenimiento.

9. Los contenidos son administrados por el usuario- administrador de manera que puede eliminar los inapropiados $\mathrm{y}$ bloquear a los usuarios que incurran en abusos.

10. La productividad en el desempeño docente se eleva porque las comunicaciones entre los maestros se mejoran. 
Entre las Redes Sociales con la tasa de crecimiento más acelerada se encuentra Google Plus, que sigue su vertiginoso ascenso entre la gente de Marketing, mientras que Twitter ha obtenido un $92 \%$ de uso con respecto al $90 \%$ de uso de Facebook, esto debido a que Twitter utiliza marcas y empresas. Otras Redes Sociales que también han incrementado su uso debido a su creciente popularidad son Pinterest e Instagram.

\section{Redes Sociales en el Campo Educativo}

Para efectos del presente estudio se estima la aplicación de las Redes Sociales: Facebook, Twitter y YouTube, como medios educativos de manera que los resultados arrojen un cuerpo de datos que contribuyan en la construcción de lineamientos teóricos para la difusión y promoción de la Educación en ámbitos informales por medio de estas redes, por lo tanto, se hace indispensable conocerlos de forma que se puedan conjugar con lo educativo para obtener sus ventajas en el proceso de enseñanza y aprendizaje para la formación del ciudadano.

\section{Red Social Facebook}

En primer lugar, se tiene la Red Social Facebook la cual fue fundada en febrero de 2004 y tiene una utilidad social que ayuda a las personas a comunicarse más eficazmente con sus amigos, familiares y compañeros de trabajo. Esta compañía desarrolla tecnologías que facilitan el intercambio de información a través del gráfico social, el mapa digital de las conexiones que existen entre las personas en el mundo real. Cualquier persona puede registrarse en Facebook e interactuar con la gente que conoce en un entorno de confianza, además forma parte de la vida de millones de personas en todo el mundo, su misión es hacer que las personas puedan compartir $\mathrm{y}$ hacer del mundo un lugar más abierto y conectado.

\section{Aplicaciones y Características}

Se pueden añadir como amigos a las personas conocidas para compartir información así como importar una lista de contactos desde un correo electrónico, también se puede utilizar para reencontrarse con personas a las que se ha perdido contacto, así mismo, se puede insertar video, música y fotos así como vincular sitios web u otras redes sociales como por ejemplo Twitter y dentro de las potencialidades para educación se tiene el chat mediante el cual se puede hacer llamada, video llamadas y enviar archivos de cualquier tipo (tales como imágenes archivos de Word, PowerPoint, Excel entre otros), hacer eventos, compartir información personal, grupos secretos, cerrados y abiertos, inclusión de archivos, entre otros.

\section{Tendencia de los Enfoques presente en los contenidos de los portales de Facebook.}

En el portal de Facebook se pueden encontrar varias aplicaciones, donde el usuario tiene a su disposición diferentes alternativas con el fin de obtener información, conocimiento, entretenimiento y poder compartirlo en tiempo real. Entre ellas se encuentra la conformación de grupos que reúnen a un conjunto de personas que tienen un interés común y forman parte del tipo de red social denominado redes sociales temáticas.

Para el presente estudio se necesita determinar unos criterios para delimitar la información presente en las redes sociales en estudio Facebook y Twitter. Por lo tanto, se consideró tomar en cuenta a los grupos denominados Grupos Ecológicos, en vista de que representan una cantidad importante de personas que se interesan por el ambiente. 
Además, es importante señalar que se observó la tendencia de los contenidos que estos grupos presentan en el portal de www.facebook.com, tomando en cuenta con una tabla de indicadores, las cuales están asociadas a las corrientes señaladas por Sauvé (2004), en la observación se encontró que en la mayoría de los contenidos se encuentran que tienen tendencia naturalista, conservacionista los cuales pertenecen a las corrientes de tendencias tradicionales.

A continuación se muestran los cuadros siguientes que muestran información relacionada con los grupos ecológicos, que utilizan la red social Facebook para su promoción, en ellos se describe el año de inicio en la red, una breve descripción de las actividades que realizan, una imagen de su portada y los enlaces correspondientes. Para la realización de los cuadros que se presentan a continuación se utilizó un baremo de indicadores de las corrientes de la Educación Ambiental según (op. cit).

\section{Cuadro 1. Tendencia Naturalista}

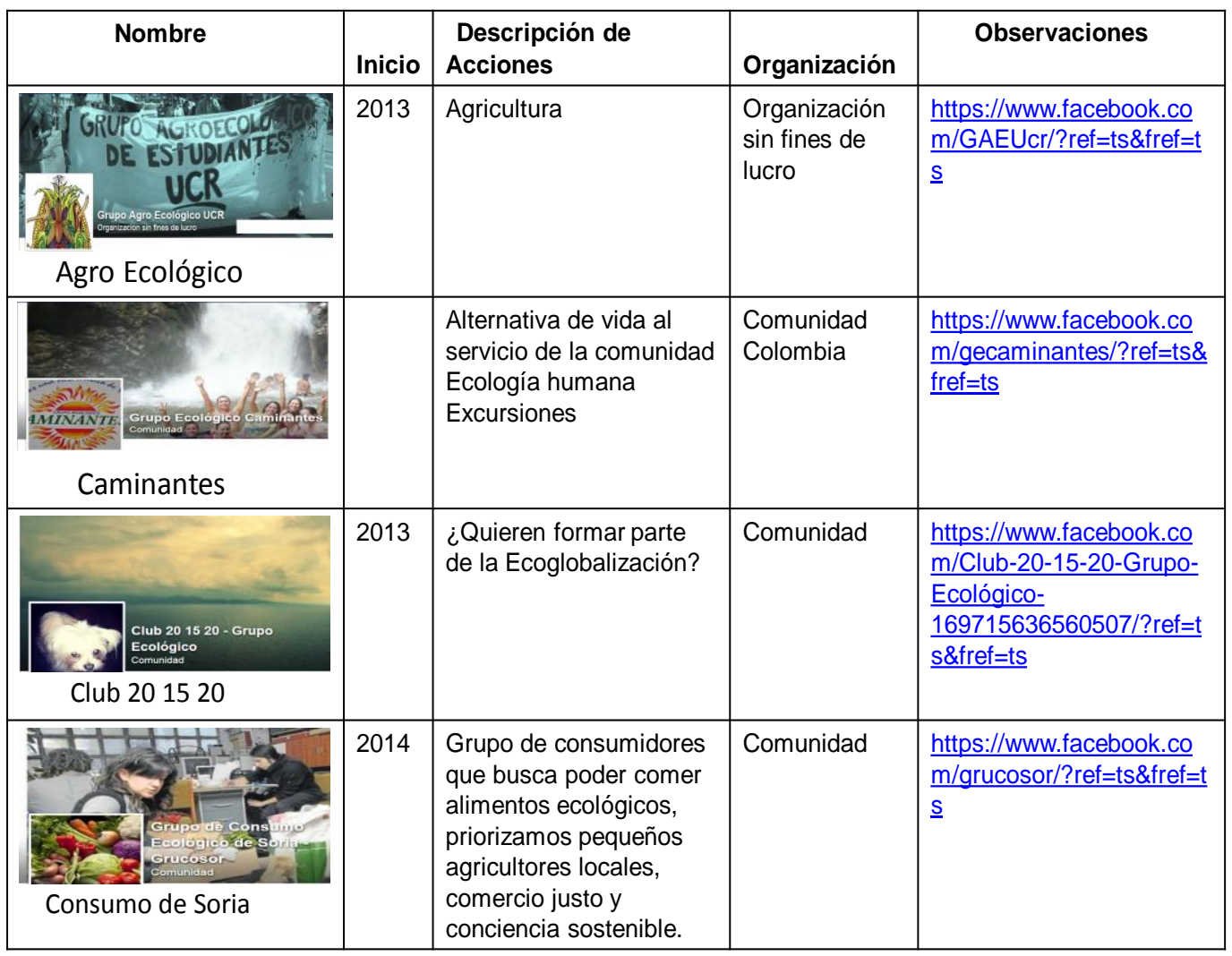


En este cuadro se reflejan las acciones que están describiendo estos portales Agro Ecológico, Caminantes Club 20 y Consumo de Soria, los cuales están más asociadas con la tendencia naturalista ya que ellos siembran y establecen el comercio y la tendencia de ganar y ganar.

También se observa la planificación de visitas a sitios naturales como ríos, playas, montañas tipo excursiones el cual corresponde con el enfoque sensualista que utiliza los sentidos para llegar a la sensación de la naturaleza en su máxima expresión para el disfrute pleno. Por otra parte, se observa la autogestión y la cogestión referente al consumo de alimentos ecológicos en agricultura local y precios justos.

En otros se reflejan las acciones donde se destaca la agricultura, el consumo agrícola, planificación de excursiones y la sensibilización ambiental. Así mismo, se puede observar que son organizaciones diversas entre las cuales están las comunidades, organizaciones no gubernamentales (ONG) y cooperativas. Se encuentra también la planificación de visitas a sitios naturales tipo excursiones así como la autogestión y la cogestión.

También se nota la presencia de grupos con tendencia conservacionista cuya característica principal es la preservación del ambiente donde se observa además que el grupo Pau Brasil tiene como lema: Conocer para amar, Amar para conservar.

Es de destacar que para contribuir con la preservación ambiental, estos grupos se han conformado solo en organizaciones comunitarias donde se manifiesta la preocupación de los miembros de esas comunidades por mantener como objetivo primordial, un ambiente sano en su entorno.

\section{Cuadro 2. Tendencia Conservacionista}

\begin{tabular}{|c|c|c|c|c|}
\hline Nombre & Inicio & $\begin{array}{l}\text { Descripción de } \\
\text { Acciones }\end{array}$ & Organización & Observaciones \\
\hline 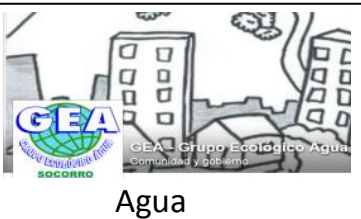 & 2013 & Comunidad-Gobierno & ONG & $\begin{array}{l}\frac{\text { https://www.facebook.com/G }}{\text { EA-Grupo-Ecológico-Água- }} \\
\text { 1425624434324290/?ref=ts } \\
\underline{\text { \&fref=ts }}\end{array}$ \\
\hline $\begin{array}{l}\text { Grupo E } \\
\text { Amantes da Natureza }\end{array}$ & 2013 & $\begin{array}{l}\text { Una ONG cuyo } \\
\text { objetivo es buscar la } \\
\text { concientización } \\
\text { ecológica como forma } \\
\text { de mejorar la calidad } \\
\text { de vida }\end{array}$ & Comunidad & $\begin{array}{l}\frac{\text { https://www.facebook.com/g }}{\text { roups/606136179490660/?r }} \\
\text { ef=ts\&fref=ts }\end{array}$ \\
\hline Ambientalista & 2012 & Siembra & Organización & $\begin{array}{l}\frac{\text { https://www.facebook.com/G }}{\text { rupoEcologicoAmbientalista/ }} \\
\underline{\text { ?ref=ts\&fref=ts }}\end{array}$ \\
\hline 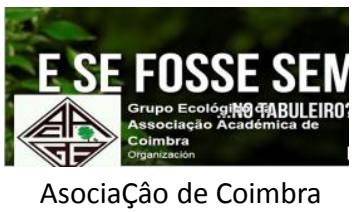 & 2012 & $\begin{array}{l}\text { Un Grupo Ecológico, } \\
\text { fundado en 1974, es } \\
\text { un espacio de debate, } \\
\text { divulgacion e } \\
\text { investigación }\end{array}$ & Organización & $\begin{array}{l}\underline{\text { https://www.facebook.com/G }} \\
\underline{\text { rupoEcologicoAAC/?ref=ts\&f }} \\
\underline{\text { ref=ts }}\end{array}$ \\
\hline
\end{tabular}


Entre los grupos ecológicos presentes en este cuadro, el grupo Agua, como organización no gubernamental (ONG), promueve la alianza entre los miembros de la comunidad y algún ente gubernamental, para llevar a cabo las acciones que se requieren para la conservación del ambiente.

También se puede notar la presencia de la comunidad organizada como es el caso del grupo "Amantes da Natureza" (sic) cuya acción es la concientización ecológica, el grupo de organización Ambientalista que promueve la siembra y el grupo Asociaçao de Coimbra que promueve la divulgación y la investigación de acciones en pro del ambiente.

Es de destacar la presencia del grupo ecológico Autónomo como una ONG que se encarga de impartir charlas en conservación del ambiente y la organización comunitaria Cartagena Joven la cual realiza jornadas de limpieza para la reducción de basura y escombros en áreas verdes. Así mismo la presencia de dos instituciones educativas que conformaron grupos ecológicos escolares para la aplicación de acciones relacionadas con la conservación del ambiente dentro y fuera de la comunidad educativa y de grupos organizacionales como: el grupo Hábitat cuyas acciones son las de idear, organizar y/o planificar y realizar acciones que permitan incluir de manera prioritaria el tema ambiental en la agenda nacional y el grupo Ne-Kié que realiza jornadas de siembra de árboles y sensibilización.

El grupo Iramuco es una causa originado por los problemas de contaminación de un lago de su comunidad y cuya acción es la de concientizar a la población de esa comunidad, acerca de la presencia de contaminación en el lago. Tenemos también el grupo comunitario Del Orituco con acciones dirigidas al reciclaje y a la sensibilización ambiental.
Los grupos ecológicos denominados San Pedro, San Vito, UNED y GE Acosta UNED, estos tres últimos se corresponden a grupos de estudiantes de comunidades universitarias, donde por una parte, se busca motivar la participación de manera voluntaria en las actividades diseñadas por estas agrupaciones y por otra parte en el ámbito educativo se caracteriza por ser un modelo integral para la formación de las futuras generaciones de líderes que puedan orientar sus acciones hacia la conservación de los recursos naturales y el desarrollo de un estilo de vida en armonía con la naturaleza.

\section{Red Social Twitter}

La Red Social Twitter es una red de información conformada por la trasmisión de mensajes en 140 caracteres llamados Tweets, el cual permite de una manera fácil y rápida tener acceso a las últimas novedades relacionadas con temas que pueda interesar a los usuarios a través de mensajes que contienen texto, fotos o videos, permitiendo obtener información exclusiva en tiempo real y acortando distancias. Dentro de las potencialidades para educación se tiene inclusión de archivos, videos, fotos, chat y algunas sugerencias para ampliar tu red y personalizarla.

A través de Twitter se pueden descubrir y seguir otras cuentas interesantes, tales como empresas, servicios públicos, celebridades, personas conocidas, fuentes de noticias. Así mismo, se puede encontrar más cuentas al ver a quienes están siguiendo las personas conocidas. En la página de inicio de Twitter aparece una secuencia de los mensajes recibidos llamada Cronología. Una vez que se sigue a personas, puntos de noticias, organizaciones o cuentas de interés personal, se tendrá una nueva página de información para leer cada vez que se inicie sesión. 
Otra propiedad es cuando se hace clic en los enlaces que aparecen en los tweets de otras personas, se pueden ver los artículos, imágenes o videos que están vinculados; también se puede etiquetar las palabras con \# cuyo nombre de esta aplicación es Hashtag, se pueden ver todos los tweets referidos sobre un tema específico, en el caso de educación a manera de ejemplo \#Filosofía \#Ortografía \#Religión y en el caso de educación ambiental se tiene \#DiadelaTierra \#DerechosHumanos \#DiadelAgua

Entre los datos de uso de Twitter se puede mencionar lo siguiente:

- 288 millones de usuarios están activos mensualmente

- Se envían 500 millones de Tweets por día

- El $80 \%$ de los usuarios activos de Twitter accede a través de su móvil.

- El $77 \%$ de las cuentas no pertenecen a usuarios de los EE. UU.

- Twitter ya se encuentra en más de 33 idiomas a nivel global.

- Tendencia de los Enfoques presente en los contenidos de los portales de Twitter

En el portal de Twitter, al igual que las otras redes sociales como Facebook y YouTube, se puede encontrar que tiene varias aplicaciones, donde el usuario tiene a su disposición diferentes alternativas para el envío de mensajes en 140 caracteres el cual le puede adjuntar fotos, videos, enlaces de páginas Web, archivos y chat, con el fin de obtener mensajes, información, conocimiento y además compartirlo en tiempo real.

$\mathrm{Al}$ igual que en la red social Facebook, se realizó un arqueo de la información y se plasmaron en una serie de cuadros para determinar algunos criterios para delimitar la información presente en la red social, se consideró tomar en cuenta, a los grupos denominados Grupos Ecológicos, en vista de que representan una cantidad importante de personas que se interesan por el ambiente.

Además, es importante señalar que se observó la tendencia de los contenidos que estos grupos presentan en el portal de www.twitter.com tomando en cuenta una tabla de indicadores, las cuales están asociadas a las corrientes señaladas por Sauvé (2004), en la observación se encontró que en la mayoría de los contenidos se encuentran que tienen tendencia naturalista, conservacionista los cuales pertenecen a las corrientes de tendencias tradicionales. En esta categoría de grupo ecológico se evidencia una presencia de grupos venezolanos, entre los que se encuentran:

@TeamParaguan4x4@PlanetariosUcab @GEA_UCV @GrupoEcologicoE @LaUrbinaVerde@ @ipceducambiente 
Cuadro 3. Cuentas en Twitter de Grupos Ecológicos

\begin{tabular}{|c|c|c|}
\hline Twitter & $\begin{array}{l}\text { Descripción de lo que } \\
\text { realizan }\end{array}$ & Enlace \\
\hline $\begin{array}{l}@ \text { TeamParaguan } 4 \mathrm{x} \\
4 \\
\text { Naturalista } \\
\text { Tweets:1314 } \\
\text { Ultimo Tw: } \\
\text { 9/3/2016 } \\
\text { Seguidores: } 1391\end{array}$ & $\begin{array}{l}\text { Un grupo sin fines de } \\
\text { lucro, ecológicos } \\
\text { amantes de la aventura } \\
\text { y lo todo terreno, } \\
\text { apasionados a los } 4 \times 4\end{array}$ & $\begin{array}{l}\text { https://twitter.com/TeamParag } \\
\text { uan4x4?lang=es }\end{array}$ \\
\hline $\begin{array}{l}\text { @PlanetariosUcab } \\
\text { Conservacionista } \\
\text { Tweets: } 64 \\
\text { Ultimo Tw: } \\
\text { 29/5/2010 } \\
\text { Seguidores: } 386\end{array}$ & $\begin{array}{l}\text { Grupo de ucabistas } \\
\text { interesados en un } \\
\text { Campus Sustentable, en } \\
\text { crear conciencia } \\
\text { ecológica y dispuestos a } \\
\text { ser el cambio a favor del } \\
\text { planeta. }\end{array}$ & 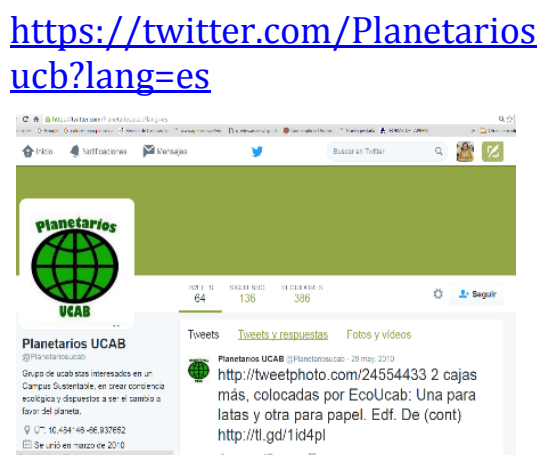 \\
\hline $\begin{array}{l}\text { @GEA_UCV } \\
\text { Conservacionista } \\
\text { Tweets: } 288 \\
\text { Ultimo Tw: } \\
\text { 15/11/2015 } \\
\text { Seguidores: } 1572\end{array}$ & $\begin{array}{l}\text { Grupo Ecológico } \\
\text { Araguaney. Escuela de } \\
\text { Estudios Políticos y } \\
\text { Administrativos - UCV. } \\
\text { ¡Conciencia Ecológica! }\end{array}$ & 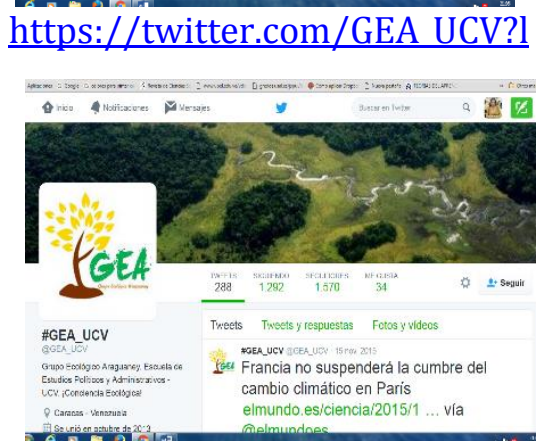 \\
\hline & & \\
\hline
\end{tabular}

\section{CONCLUSIONES}

La Educación Ambiental formal, no formal e informal a través de las redes sociales reciente y poco estudiando por la mayoría de docentes, administradores y planificadores educativos lo que genera una normal desconfianza, resistencia al cambio y poco interés por capacitarse, formase y poner en práctica sus conocimientos al servicio de la educación.

Sin embargo se considera que puede ser una gran herramienta en la promoción, divulgación de la educación ambiental, porque permite diversas formas de comunicación y rompe las barreras de espacio y tiempo.

La educación ambiental ha adquirido una especial importancia hoy en día a raíz de la crisis ambiental que atraviesa el planeta a causa de las acciones humanas no cónsonas con el entorno natural.

Es imprescindible concientizar $y$ sensibilizar a la familia, a la comunidad, a la sociedad, a la humanidad por medio de un equipo multidisciplinario que de manera perseverante muestre las alternativas de 
solución educativa a la falta formación del ciudadano ante las situaciones ambientales.

\section{REFERENCIAS}

Agenda 21 (1992). Capitulo 36. Fomento de la Educación, la Capacitación y la Toma de Conciencia. [Documento en línea] Disponible:

http://www.un.org/spanish/esa/sutdev/ agenda21spchapter36.htm [Consulta: 2019, Marzo 13]

Ballestrini, F., Marcon, A., Morduchowicz, R. y Sylvestre, V. (2010). Los Adolescentes y las Redes Sociales. Ministerio de Educación Presidencia de la Nación. Escuelas y Medios. Disponible en: http://www.me.gov.ar/escuelaymedios/ material/redes.pdf [Consulta 2019, Marzo 13]

Beltrán y Castañeda (2004), en Moreno, R. y Castellanos, S. (2003). "Definición de un Modelo de Redes de conocimiento como soporte a la transferencia de conocimiento generado en cluster de investigación" en Revista del Centro de Innovación y Desarrollo para la Investigación en Ingeniería del Software CIDLIS-.

Bonnelly, R. (2011). La Huella Social. Libros de El Nacional. Editorial Arte. Caracas. Venezuela

Burgueño, P. (2009). Clasificación de redes sociales. Recuperado el 04 de marzo de 2019, http://www.pabloburgueno.com/2009/0 3/clasificacion-de-redessociales

Bucarelo, M. y Zambrano, F., (2010). Las Redes Sociales Disponible: http://rsucatinformatica.blogspot.com/p /clasificacion-de-las-redes-sociales.html [Consulta: 2019, marzo 10]

Chópite, C. (2014). Mercadeo en redes sociales para el posicionamiento de empresas de equipamiento de cocina del municipio Maracaibo. Marketing Visionario, 3(1), 2-19.

Coordinadora de ONG para el Desarrollo de España (2004). Educación para el Desarrollo: Estrategia Imprescindible para el Desarrollo. Propuestas para el
Plan Director 2005-2008. [Documento en línea] Disponible: http://www.educacionenvalores.org/IM G/pdf/queeslaED.pdf [Consulta: 2012, Octubre 12]

De Haro, J. J. (2008). Mapa conceptual: Aplicaciones educativas de las redes sociales. jjdeharo. blogspot. com. es. Extraído el,13. Disponible en: http://jjdeharo.blogspot.com/2008/11/l a-redes-sociales-en-educacin.html

De Marco, A. (2008). Reseña de libro: Pedagogía e Internet. Aprovechamiento de las Tecnologías. Autores: Armand StPierre y Natalie Kustcher. México. Editorial Trillas. Año: 2001. Revista de Investigación. № 65. 296-297.

De Marco, A. (2015). La Autorregulación Del Aprendizaje En La Modalidad Mixta En La Universidad Pedagógica Experimental Libertador- Instituto Pedagógico de Caracas (Upel-Ipc) Una Propuesta Teórica para su Implementación. Tesis Doctoral no publicada, UPEL-IPC, Caracas.

Faundes, C y Aristizabal, C (2001). Era Cibernética: La Revolución Digital. [Documento en línea] Disponible en: http://www.terra.com/especiales/revolu ciondigital/eracibernetica.html [Consulta: 2019, marzo 20]

Fernández, E. (2010). Marketing 2.0 en una Semana. España. Editorial Centro Libros PAPF

Fernández, E. (2019). El uso de las TAC en el nivel de concentración y participación en la materia Emprendimiento $y$ Gestión (Bachelor's thesis, Universidad de Guayaquil. Facultad de Filosofía, Letras y Ciencias de la Educación)

González, L., Ureta, L., Marcovecchio, M., Margarit, V., Pontoriero, F., Rossetti, G., Villodre, S. (2019) Alfabetización con formatos múltiples de aprendizaje: las TAC $\mathrm{y}$ los nuevos escenarios tecnopedagógicos en la universidad. Disponible en: http://sedici.unlp.edu.ar/handle/10915/ 77319

Guzmán, B. (2005). Actitudes de los docentes ante las Tecnologías de Información y 
Comunicación Tesis Doctoral no publicada UPEL-IPC. Caracas.

Guzmán, B. (2008). Los docentes de la Universidad Pedagógica Experimental Libertador - Instituto Pedagógico De Caracas ante las Tecnologías De Información Y Comunicación Investigación y postgrado, ISSN 13160087, 1(23), págs. 37-72 Disponible en $<$ http://ve.scielo.org/scielo.php?script=s ci_arttext\&pid=S131600872008000100003\&lng=es\&nrm=iso> . [Consulta: 2019 marzo 16].

Guzmán, B. (2013). Evaluación del Curso Tecnología de Información y Comunicación y Educación Ambiental a Través de sus Productos. Trabajo de ascenso. Instituto Pedagógico de Caracas.

Guzmán, B. (septiembre 2018) TIC y Medios. 27 Conferencia presentada en Encuentro Nacional de Profesores de Francés organizado y promovido por la Asociación Venezolana de Profesores de Francés. Pozo de Rosas, Miranda.

Guzmán, B. (2019) TIC y Visualización de los resultados Conferencia presentada en Encuentro Nacional Saberes e investigación Ciudadela Maracaibo.

Hobrayan, M., Carpio, Z., y Villagómez, A., (2019): "La Innovación Tecnológica (TIC y TAC) en una escuela de educación primaria", Revista de Investigación Latinoamericana en Competitividad Organizacional RILCO, n. 2 (mayo 2019). Disponible en: https://www.eumed.net/rev/rilco/02/ti c-tac.html http://hdl.handle.net/20.500.11763/rilc o02tic-tac [Consulta: 29/10/2019]

Jaramillo, A. (2011) Redes Sociales para Todos. Su negocio en la Web 2.0.Editorial Vergara. Ediciones B Colombia S.A.

Krotoski, A (2012, September 11) Virtual Revolution: E4. WOBI Channel. World of Business Ideas. London

Lárez, H. y Carrero, A. (2003) Etnografía: posibilidades de uso en la Investigación en Educación Ambiental. Revista de Investigación. № 54. 109-134.Caracas
López, E. (2016). Teoría Subyacente en la Promoción y Divulgación de la Educación Ambiental Informal para la Formación del Ciudadano por medio de las Redes Sociales Digitales. Tesis Doctoral no publicada, Universidad Pedagógica Experimental Libertador, Instituto Pedagógico de Caracas.

Marquina, R. (2011, Julio). Hacia una Docencia 2.0. Realidades y Mitos. Ponencia presentada en el 1er. Encuentro Nacional Ciudades Digitales. Chacao, Caracas.

Martínez, M. (2010). Teoría Emergente. Editorial Trillas.

Medina, S. De Haro Ollé, J. J. (2010). Redes sociales para la educación. Madrid: Anaya Multimedia, 432 pp. Estudios sobre Educación, 20, 262.

Rodríguez, W (2010) Estado del Arte Redes Sociales en Educación Corporación Universitaria Minuto de Dios. Facultad de Educación. Bogotá Disponible en: http://hdl.handle.net/10656/615 [Consulta: 2019 marzo 16].

Sauvé, L. (2004). Una cartografía de corrientes en Educación Ambiental. Cátedra de investigación de Canadá en educación ambiental. La investigación en Educación Ambiental. Université du Québec à Montréal. Disponible en: http://www.ambiente.gov.ar/infotecaea/ descargas/sauve01.pdf. [Consulta: 2019, marzo 16].

Sevillano, M. (2005). Didáctica en el Siglo XXI. McGraw Hill/InterAmericana de España. S.A.U. España

Sobrino, A. (2011). Proceso de enseñanzaaprendizaje y web 2.0: valoración del conectivismo como teoría de aprendizaje post-constructivista. [Documento en línea].

Disponible:http://ddigital.umss.edu.bo:8 080/jspui/bitstream/123456789/13

80/1/conectivismo.pdf [Consulta: 2016, Abril 10]

Suarez, V (2012, Septiembre 23) Internet en América Latina. El Universal, Cuerpo 1, p. 9. Economía 